\title{
Learning about Galactic structure with Gaia astrometry
}

\author{
Anthony G.A. Brown \\ Sterrewacht Leiden, Leiden University, P.O. Box 9513, 2300 RA Leiden, The Netherlands
}

\begin{abstract}
The Gaia mission is reviewed together with the expected contents of the final catalogue. It is then argued that the ultimate goal of Galactic structure studies with Gaia astrometry should be to build a dynamical model of our galaxy which is capable of explaining the contents of the Gaia catalogue. This will be possible only by comparing predicted catalogue data to Gaia's actual measurements. To complement this approach the Gaia catalogue should be used to recalibrate photometric distance and abundance indicators across the HR-diagram in order to overcome the lack of precise parallax data at the faint end of the astrometric survey. Using complementary photometric and spectroscopic data from other surveys will be essential in this respect.
\end{abstract}

Keywords: Astrometry, Galaxy: kinematics and dynamics, Galaxy: formation, Galaxy: structure PACS: 95.55.Br, 95.80.+p, 98.35.Ac, 98.35.Ce, 98.35.Df

\section{OVERVIEW OF THE GAIA MISSION}

Gaia will provide a stereoscopic census of our Galaxy through the measurement of high accuracy (global and absolute) astrometry, radial velocities and multi-colour photometry. Gaia will measure parallaxes and proper motions for every object in the sky brighter than magnitude 20 - amounting to over 1 billion stars, galaxies, quasars and solar system objects. It will achieve a complete all-sky survey to its limiting magnitude via real-time on board detection. The astrometric accuracy will be 12-25 $\mu$ as, depending on colour, at 15 th magnitude and 100-300 $\mu$ as at 20th magnitude. Multi-colour photometry will be obtained for all objects by means of low-resolution spectrophotometry. The photometric instrument consists of two prisms dispersing all the light entering the field of view. One disperser - called BP for Blue Photometer - operates in the wavelength range 330-680 nm; the other - called RP for Red Photometer - covers the wavelength range $640-1050 \mathrm{~nm}$. In addition radial velocities with a precision of $1-15 \mathrm{~km} / \mathrm{s}$ will be measured for all objects to 17 th magnitude, thus complementing the astrometry to provide full six-dimensional phase space information for the brighter sources. The radial velocity instrument (RVS) is a near-infrared ( $847-874 \mathrm{~nm}, \lambda / \Delta \lambda \sim 11000)$ integral-field spectrograph dispersing all the light entering the field of view. Gaia builds on the proven principles of the Hipparcos mission but represents an improvement of several orders of magnitude in terms of numbers of objects, accuracy and limiting magnitude (Hipparcos observed 120000 stars to 12th magnitude, achieving milli-arcsecond accuracy).

The Gaia satellite and mission operations are fully funded by the European Space Agency and the launch is foreseen in December 2011. Gaia will orbit the Sun-Earth L2 Lagrange point at 1.5 million $\mathrm{km}$ from the Earth. The transfer to this point and the commissioning of the spacecraft and payload will take up to 6 months, after which 
the routine science operations start. This phase will last 5 years, with a possible 1 year extension. The data processing will be undertaken by the scientific community in Europe which has organized itself into the Gaia Data Processing and Analysis Consortium (DPAC). The processing will be ongoing during the operational phase and for 2-3 years after. The final catalogue is expected around 2021, but with intermediate data releases produced during the operational phase.

In the context of Galactic structure studies it is interesting to consider the information which the final Gaia catalogue will contain on stars. In Gaia's own broad-band magnitude $G$ the number of stars will be $\sim 7 \times 10^{5}$ to $G=10,48 \times 10^{6}$ to $G=15$ and $1.1 \times 10^{9}$ to $G=20$. About 60 million stars are expected to be seen as binary or multiple systems by Gaia. For each star the following information is provided:

astrometry positions, parallax, proper motions, the full covariance matrix of the astrometric parameters (standard errors and correlations) and astrometric solution quality indicators.

photometry broad band fluxes in the $G, G_{\mathrm{BP}}, G_{\mathrm{RP}}$ and $G_{\mathrm{RVS}}$ bands, as well as the prism spectra measured by the blue and red photometers. Variability indicators will be provided for all stars together with epoch photometry.

spectroscopy the radial velocity is listed for each star observed by RVS and for the brighter stars $v \sin i$ as well as the accumulated spectra will be provided.

multiple stars solution classifications and, where relevant, orbital parameters together with covariance matrices and quality flags.

astrophysical parameters the Gaia catalogue will provide as much astrophysical information on each star as possible, derived from the combination of photometric, spectroscopic and parallax information. The astrophysical parameters include $T_{\text {eff }}$, $A_{V}, \log g,[\mathrm{M} / \mathrm{H}]$, and $[\alpha / \mathrm{Fe}]$ where possible. Luminosities, ages, and variable star characterizations will also be provided.

The catalogue will also contain intermediate data which allow a reprocessing of the observations. In particular the astrophysical parameters can be improved by incorporating complementary information obtained from other surveys or follow-up observations. For astrometric data reprocessing is relevant for multiple sources with uncertain solutions (which can be improved by incorporating follow-up radial velocity observations) or, for example, in the case of proper motion measurements for faint stars in a dwarf galaxy orbiting the Milky Way one could combine the measurements for all stars to derive a (more precise) mean motion and parallax for the dwarf. Hence the Gaia catalogue will not be a static release but will evolve over time as more information is added to it.

For much more information on the Gaia mission and the science topics that will be addressed I refer to the proceedings of the symposium The Three-Dimensional Universe with Gaia [1] and recent overviews of the mission status [2] and DPAC [3].

\section{GALACTIC STRUCTURE STUDIES WITH GAIA}

Before highlighting some of the Galactic structure topics where Gaia's astrometric data is expected to have a major impact it is useful to consider its astrometric performance in 
relation to other large existing or upcoming surveys of the Galaxy (see [4] and [5]). From this comparison it is clear that Gaia will be the only mission in the coming decade that provides astrometry in the 10-100 $\mu$ as regime in the optical for large all-sky samples and covering a large volume throughout our Galaxy. Important complementary astrometry will be provided by the JASMINE mission in the near infrared (covering the bulge and inner disk regions), the SIM Planetquest mission in the optical to accuracies of a few $\mu$ as for selected targets, and by the LSST and Pan-STARRS surveys which will provide milli-arcsecond astrometry to 24th magnitude.

Some of the top questions concerning our galaxy are listed in [5]. What is the mass distribution throughout the Galaxy? What is the spiral arm structure? What is the merging history of our galaxy? How many mergers occurred and what did the building blocks look like? Is our galaxy consistent with the $\Lambda$ CDM paradigm? How and when were the structural components (bulge, halo, thin/thick disk) of the Galaxy assembled? Which stars formed where and when did they form? The most direct impact of Gaia astrometry on these questions will be through the vast amount of stars for which accurate 5-dimensional phase space data will be available. Indirectly the astrometric data, in particular the parallaxes, will enable a highly accurate mapping of the Hertzsprung-Russell diagram, thus tremendously improving our understanding of stars and their atmospheres. As a consequence the astrophysical characterization of stars will be improved leading to better age and chemical composition determinations. This enables a detailed reconstruction of the evolution of the Galaxy and its structural components in time. A few examples of Galactic structure studies with Gaia are discussed in some more detail below. Much more examples and extensive discussions can be found in [5].

In a large volume around the Sun the space motions of stars will be accurately determined (using the RVS data) which allows the reconstruction of their orbits and the tracing of their birthplaces in the Galaxy. When complemented by precise stellar ages and the possibility to construct volume limited samples for spectroscopic followup studies, the detailed reconstruction of the history of the disc becomes possible by disentangling the effects of stars formation history, dynamics, and chemical evolution of the interstellar medium on the observed properties of stars.

The stellar halo was largely built up through accretion and/or mergers and a major goal is to identify the building blocks. Recently many tidal streams and dwarf satellites orbiting our Galaxy have been identified, in large part due to the Sloan Digital Sky Survey. However these objects are mostly confined to the outer halo and the analysis of stellar abundances for dwarf satellites and field halo stars make it clear that these newly discovered streams and dwarfs are not the building blocks of the bulk of the halo. The accretion history of the halo is thus to a large extent contained in its field stars and tracing these to the original building blocks is difficult as they have been thoroughly mixed in configuration space. Gaia is uniquely capable of providing the necessary phase space information for the inner halo which is needed in combination with the astrophysical information on the stars in order to reconstruct the formation history of the halo.

Finally, Gaia will tremendously advance the mapping of the spiral structure of our galaxy. A direct tracing of spiral arms is possible through the accurate distance information for early type stars. Indirectly the arms can be traced through the stellar kinematics in the disk. Gaia parallaxes are also required for the construction of a 3D extinction map which is needed to overcome the obscuring effects of dust in the plane of the Milky Way. 


\section{FROM GAIA TO A DYNAMICAL MODEL OF THE GALAXY}

Studies of specific Galactic components are important but do not provide the whole picture. The structural components are coupled through gravity and the observed stellar and gas kinematics are determined by the gravitational potential of the Galaxy. The only way to develop a consistent understanding of the mass distribution and kinematics is through a dynamical model of the Galaxy, and it is only with such a model that one can make reliable extrapolations to the unobserved parts of Galactic phase space. The Gaia catalogue can be seen as a snapshot of the state of the Galaxy in which we will be seeing stars from the same population at different points along the same orbits. This allows the reconstruction of individual orbits from which we can infer the Galactic potential and matter distribution. Any dynamical model will thus be highly constrained.

Hence as argued in [6], if we want to take full advantage of an all-sky high accuracy astrometric data-set (complemented by radial velocities, photometry and astrophysical information) and convert this data for 1 billion stars into a complete physical understanding of the structure of our galaxy, the goal should really be to construct a dynamical model in terms of which we can explain the entire Gaia catalogue. This is obviously a non-trivial task and I discuss below the steps that should be taken in anticipation of the Gaia catalogue and once it is available.

\section{Dynamical modelling techniques}

Constructing such a dynamical model is a difficult task as it has to be able to selfconsistently determine matter and velocity distributions from the underlying potential. Moreover, in comparing with the Gaia catalogue data the astrophysical properties of the stellar populations (composition, age) as well as the effects of extinction due to dust have to be accounted for. A dedicated programme to prepare such models is therefore needed as described in [6]. The latter paper describes three promising approaches to the construction of the required Galaxy model; extensions of the Schwarzschild method, made-to-measure N-body techniques, and the torus technique.

The Schwarzschild method [7] has been applied extensively to external galaxies in order to derive their phase space structure from observations of the line of sight velocity distributions or integral field spectrograph data. The method works by calculating an orbit library in a trial potential and then finding the weighted superposition of orbits which can reproduce continuous observables such as surface brightness and velocity moments. The application to Gaia data requires extending the method so it can be used with discrete data for individual stars. A first step in this direction has been taken in [8] but the method will have to be extended to triaxial geometries and has to be capable of dealing with effects such as that of the Milky Way's bar.

The made-to-measure N-body method was introduced in [9] and applied to the Milky Way as described in [10]. Unlike the Schwarzschild method this technique does not pre-calculate large libraries of orbits followed by a weighted superposition. Rather, the weights are determined simultaneously with the integration of the orbits. This method can be incorporated into N-body simulations (e.g., to generate equilibrium initial con- 
ditions for large- $N$ systems) and is flexible in that it allows for arbitrary geometries and potentials that evolve in time. The challenge will be to overcome the computational complexity involved when trying to reproduce the Gaia catalogue data.

Finally, the torus technique [11] replaces the orbit library used in the Schwarzschild method with phase-space tori. This method requires complex software and has so far only been demonstrated for axisymmetric systems. In addition the orbits are derived in an integrable potential while the true potential of the Milky Way is certainly not integrable. The torus method requires extensive development in order to be able to deal with realistic Milky Way potentials. Some ideas are given in [6].

The development of these techniques should start now but we do not have to wait for the Gaia catalogue to be finished before applying them, as there will be plenty of surveys providing photometric and kinematic data for large samples of stars in our galaxy.

\section{Finding the best Galaxy model}

As noted in [6] any of the methods just described will lead to a dynamical model of the Galaxy consisting of a gravitational potential and distribution functions for each of the stellar populations. The latter are described as probability distributions in mass, chemical composition and age for the stars in a particular population. Hence the Galaxy model will consist of a very large number of parameters and 'fitting' the model to the entire Gaia catalogue will be a very challenging task.

The basic predictions from the models are the distributions of the stars in phase-space $(\mathbf{r}, \mathbf{v})$ at some time. These distributions could be compared to phase-space variables calculated from the Gaia astrometric and radial velocity data in order to decide on the best Galaxy model. This approach suffers from the fact that for most stars the radial velocity will not be available, but more importantly it requires the transformation from observed parallaxes to distances. It is important to keep in mind that we do not directly measure the distance to stars. What we measure instead are their parallactic displacements on the sky caused by the motion of the earth around the sun. The transformation of the observed parallax $\varpi_{o}$ to distance, given by $r_{o}=1 / \varpi_{o}$, is seemingly trivial but can cause many problems because it is non-linear. In the absence of systematic measurement errors the observed parallax is itself an unbiased estimate of the true parallax $\varpi: E\left[\varpi_{o}\right]=\varpi$. This is not true of the distance, i.e. $E\left[1 / \varpi_{o}\right] \neq 1 / \varpi$. This well-known problem was discussed at the time of the release of the Hipparcos Catalogue [12] and plays an important role in luminosity calibrations where effects of the Lutz-Kelker type can lead to biased estimates of the absolute magnitudes of stars. The same problems occur when calculating the positions and motions of stars (all proportional to $1 / \varpi$ ) or integrals of motion such as energy $E$ or angular momentum $L$ (both functions of $1 / \varpi^{2}$ ). The energy-angular momentum plane is is a powerful tool when looking for remnants of accreted satellites. However as shown in [13] the propagation of parallax errors can lead to sign changes in

$L_{z}$, spurious features in the $E-L_{z}$ plane, and in addition the $1 / \varpi^{2}$ dependence will cause the estimates of the integrals of motion for a particular satellite to be biased.

The only truly robust way to get around this problem is to project the Galaxy model into the data-space and thus predict the astrometric data together with the other data 
in the Gaia catalogue (radial velocities, magnitudes and colours of stars). The added advantage is that one can easily account for incomplete phase space data (e.g., lack of radial velocity data) and selection effects. The extinction due to dust can be taken into account in predicting the observed distribution of magnitudes and colours of the stars. Moreover, negative parallaxes (which are perfectly legitimate measurements!) and the correlations in the errors on the astrometric parameters (which will vary systematically over the sky) can be much more easily accounted for in the data-space.

To decide on the best values for the Galaxy model parameters one would ideally use the maximum likelihood technique. However, given the complications of the Galaxy model and the large number of parameters it will very likely not be possible to construct the likelihood function and its derivatives (need for its maximization). One will therefore have to resort to generating mock Gaia catalogues from the model and comparing these to the actual data. The challenges here are the comparison of predicted and observed distributions of observables for very large amounts of data and the exploration of a very high dimensional model space in order to find the optimum parameter values. The result should be a probability distribution over the model space (where we should keep in mind that not all aspects of the Galaxy model will be uniquely determined). The problem of finding this distribution for a very high dimensional parameter space is a well known issue in inverse problem theory [14]. Many efficient methods for exploring the parameter space have been developed over the years, such as Markov Chain Monte Carlo methods, and our quest for a dynamical model of the Milky Way can take advantage of this knowledge. An extensive discussion on how the Torus technique can be combined with the inverse problem methodology is given in [15] for the case of spherical systems.

\section{Gaia alone is not enough}

To get the dynamical modelling effort started a direct examination of the positions and kinematics of the stars will still be essential. This will enable us to characterize the structural components of the Galaxy, the degree of non axisymmetry, and the amount and kind of substructure present in the halo. The identification and description of Galactic stellar populations will provide further constraints on the model (see [15]) and is essential to the study of Galactic structure as a function of time.

In order to do this accurate alternative distance indicators are needed for the faint and distant stars in the Gaia survey to supplant their relatively imprecise parallaxes. This will also enhance the separation and description of stellar populations through better determined luminosities, ages, and chemical compositions. Gaia will enable us to recalibrate to high accuracy photometric distance indicators across the HR-diagram. To illustrate this Table 1 shows Gaia's reach in terms of relative parallax precision for a selected set of stellar types.

Precise photometric distance indicators cannot be derived from the Gaia data alone as at the faint end the determination of extinctions, surface gravities and abundances from Gaia's photometry will not be reliable. Complementary data from other large area surveys such as Pan-STARRS, LSST, and SkyMapper, as well as follow-up spectroscopic observations, are needed to calibrate photometric abundance and $\log g$ indicators. 
TABLE 1. Parallax horizon of Gaia. This is the maximum distance (in $\mathrm{kpc}$ ) at which a given relative precision in parallax (or distance) is obtained. Based on latest accuracy estimates, with $M_{V}$ from [16]. Table reproduced from [2].

\begin{tabular}{lcccccccccc}
\hline & \multicolumn{3}{c}{ No extinction $\left(A_{V}=0\right)$} & & \multicolumn{4}{c}{$A_{V}=5 \mathrm{mag}$} \\
\cline { 2 - 7 } \cline { 8 - 11 } Type of star & & & & & & & & & & \\
\hline G0V $\left(M_{V}=+4.4\right)$ & 0.8 & 1.1 & 1.8 & 2.5 & & 0.3 & 0.5 & 0.7 & 1.0 \\
K5III $\left(M_{V}=-0.1\right)$ & 1.3 & 2.6 & 4 & 7.5 & & 1.0 & 1.5 & 2.4 & 3.5 \\
Cepheid $\left(P=10^{\mathrm{d}}, M_{V}=-4.1\right)$ & 1.2 & 2.4 & 6 & 12 & & 1.2 & 2.3 & 3.8 & 7 \\
\hline
\end{tabular}

Wide-field multi-object spectrographs covering the UV/blue, deployed on 4 and 8 meter class telescopes, will be essential in this respect. The complementary photometric data in combination with data at longer wavelengths is also needed to determine precise extinction values for stars in the Gaia catalogue.

In conclusion, the Gaia mission will provide a vast and highly accurate astrometric data-set, complemented by astrophysical information, which offers a fantastic opportunity to obtain a complete physical understanding of our Galaxy. We should start preparing now for the challenge of building the required dynamical model.

\section{REFERENCES}

1. C. Turon, K. S. O'Flaherty, M. A. C. Perryman, The Three-Dimensional Universe with Gaia, ESA SP-576, 2005.

2. L. Lindegren, et al., "The Gaia mission: science, organization and present status", in A Giant Step: From Milli- to Micro- Arcsecond Astrometry, edited by W. J. Jin, I. Platais and M. A. C. Perryman, Proceedings IAU Symposium No. 248, Cambridge University Press, Cambridge, 2008, pp. 217-223.

3. F. Mignard, et al., "Gaia: organisation and challenges for the data processing", in A Giant Step: From Milli- to Micro- Arcsecond Astrometry, edited by W. J. Jin, I. Platais and M. A. C. Perryman, Proceedings IAU Symposium No. 248, Cambridge University Press, Cambridge, 2008, pp. 224-230.

4. A. G. A. Brown, "Getting ready for the micro-arcsecond era", in A Giant Step: From Milli- to MicroArcsecond Astrometry, edited by W. J. Jin, I. Platais and M. A. C. Perryman, Proceedings IAU Symposium No. 248, Cambridge University Press, Cambridge, 2008, pp. 567-576.

5. C. Turon, et al., Galactic Populations, Chemistry and Dynamics, ESA-ESO working groups Report no. 4, 2008, available at www.stecf.org/coordination/eso-esa/galpops.php

6. J. Binney, "Modelling the Galaxy for Gaia", in The Three-Dimensional Universe with Gaia, edited by C. Turon, K. S. O’Flaherty and M. A. C. Perryman, ESA SP-576, 2005, pp. 89-95.

7. M. Schwarzschild, ApJ 232, 236-247 (1979).

8. J. Chanamé, J. Kleyna, R. van der Marel, ApJ 682, 841-860 (2008).

9. D. Syer, S. Tremaine, MNRAS 282, 223-233 (1996).

10. N. Bissantz, V. P. Debattista, O. Gerhard, ApJ 601, L155-L158 (2004).

11. W. Dehnen, J. Binney, "Dynamical Models of the Milky Way", in Formation of the Galactic Halo...Inside and Out, edited by H. Morrison and A. Sarajedini, ASP Conference Series, Vol. 92, 1996, pp. 393-402.

12. A. G. A. Brown, F. Arenou, F. van Leeuwen, L. Lindegren, X. Luri, "Some considerations in making full use of the Hipparcos Catalogue", in Hipparcos Venice '97, ESA SP-402, 1997, pp. 63-68

13. A. G. A. Brown, H. M. Velázquez, L. A. Aguilar, MNRAS 359, 1287-1305 (2005).

14. A. Tarantola, Inverse problem theory and methods for model parameter estimation, Society for Industrial and Applied Mathematics, Philadelphia, 2005.

15. M. Kaasalainen, Inverse problems and imaging, astro-ph/0809.0218, (2008).

16. A. N. Cox, Allen's astrophysical quantities, 4th ed., AIP press, Springer New York, 2000. 